\title{
Influence of the crystallographic and the morphological texture on the elastic properties of fcc crystal aggregates
}

\author{
K. Jöchen a, T. Böhlke ${ }^{b}$ and F. Fritzen ${ }^{c}$ \\ Institute of Engineering Mechanics, Chair for Continuum Mechanics, Karlsruhe Institute of \\ Technology (KIT), P.O. Box 6980, D-76128 Karlsruhe, Germany \\ a joechen@itm.uni-karlsruhe.de, b boehlke@itm.uni-karlsruhe.de, c fritzen@itm.uni-karlsruhe.de
}

Keywords: self-consistent homogenization, crystallographic texture, morphological texture

\begin{abstract}
In this contribution, the prediction of the self-consistent homogenization method with regard to the effective material response of cubic crystal aggregates is analyzed and compared to results from full field simulations. The influence of the crystalline orientation distribution but also the effect of the grain shape on the macroscopic elastic response of sheet metals is especially emphasized.
\end{abstract}

\section{Introduction}

The macroscopic material behavior of crystalline materials is significantly affected by the constitutive behavior of the grains but also by the crystallographic and morphological texture. To accurately predict the global material response of crystalline aggregates, detailed microstructural information has to be incorporated into the evaluation scheme. In this paper, we analyze one-point bounds and the self-consistent scheme with regard to the effective material behavior of sheet metals consisting of cubic single crystals. The results are compared to finite element based predictions for the effective response. The crystal orientation distribution can be taken into account by all three approaches, whereas it is only possible to study the influence of the grain morphology by the selfconsistent method and the numerical homogenization scheme because the one-point bounds are insensitive to morphological details.

\section{Crystallographic and morphological texture of rolled sheets}

In the rolling process of polycrystalline aggregates to produce sheet metals, the grains of the polycrystal undergo severe deformations. Due to this process, the grain orientations change significantly and also the grain shape becomes highly anisotropic. The crystallite orientation distribution can be described by a distribution function $f(\boldsymbol{Q})$ (codf) specifying the volume fraction $d v / v$ of crystals with the orientation $\boldsymbol{Q}$, where $\boldsymbol{Q}$ is an orthogonal second-order tensor.

In this study, we create the initial codf in the rolling process by a rigid-viscoplastic Taylor-type simulation [1], i.e., assuming a homogeneous deformation in the whole crystal aggregate

$$
\overline{\boldsymbol{F}}=\lambda \boldsymbol{e}_{R} \otimes \boldsymbol{e}_{R}+\lambda^{-1} \boldsymbol{e}_{N} \otimes \boldsymbol{e}_{N}+\boldsymbol{e}_{T} \otimes \boldsymbol{e}_{T} .
$$

The vectors $\boldsymbol{e}_{R}, \boldsymbol{e}_{N}, \boldsymbol{e}_{T}$ are orthogonal unit vectors in the rolling, normal and transverse direction of the sheet metal, respectively. Starting with 1000 uniformly distributed grain orientations and by applying different degrees of thickness reductions $\lambda^{-1}$ in the rolling process, the Taylor model yields rolling textures as depicted by the pole figures in Fig. 1, which overestimate the texture sharpness. The mean deformation gradient (Eq. 1) applied to run the Taylor-simulations is also used to characterize the mean grain shape of the grains forming the sheet metal. 

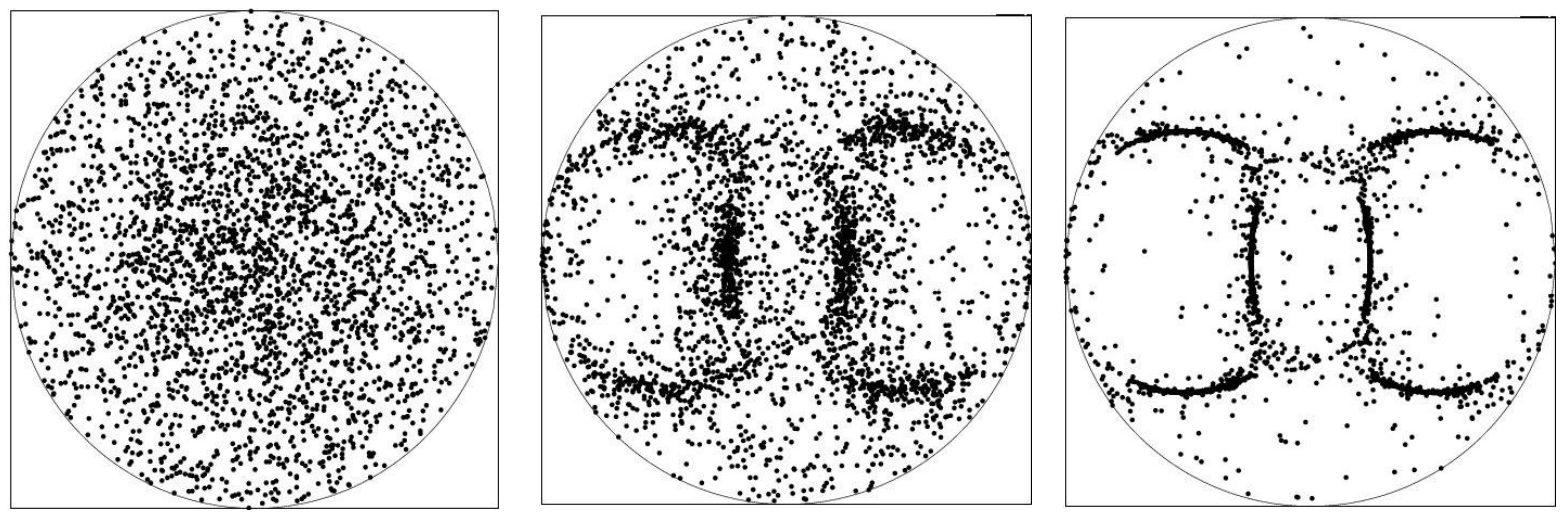

Fig. 1: <100>-pole figures of the rolling texture of 1000 grains obtained by Taylor-type simulations: $\lambda=1$, i.e., $0 \%$ thickness reduction (left); $\lambda=2$, i.e., $50 \%$ reduction (middle): $\lambda=10$, i.e., $90 \%$ reduction (right).

\section{Effective elastic properties}

First-order bounds. The simplest approach to predict the effective elastic behavior of polycrystalline aggregates is to use the arithmetic and harmonic mean of the local stiffness tensors. The arithmetic and harmonic mean, first suggested by Voigt and Reuss, correspond to the assumption of homogeneous strain and stress fields, respectively. These approaches provide upper and lower bounds for the strain energy density and can be written as

$$
\mathbb{C}^{V}=\int_{S O(3)} f(\boldsymbol{Q}) \mathbb{C}(\boldsymbol{Q}) \mathrm{d} Q=\int_{S O(3)} f(\boldsymbol{Q}) \boldsymbol{Q} \star \tilde{\mathbb{C}} \mathrm{d} Q
$$

and

$$
\mathbb{S}^{R}=\int_{S O(3)} f(\boldsymbol{Q}) \mathbb{S}(\boldsymbol{Q}) \mathrm{d} Q=\int_{S O(3)} f(\boldsymbol{Q}) \boldsymbol{Q} \star \tilde{\mathbb{S}} \mathrm{d} Q
$$

Here, $\tilde{\mathbb{C}}$ and $\tilde{\mathbb{S}}$ Fehler! Textmarke nicht definiert.denote the reference stiffness and compliance tensor, respectively. The $*$ denotes the Rayleigh product, which for tensors of arbitrary rank $\mathbb{T}=$ $T_{\mathrm{ij} \ldots 1} \boldsymbol{e}_{\mathrm{i}} \otimes \boldsymbol{e}_{\mathrm{j}} \otimes \ldots \otimes \boldsymbol{e}_{1}$ is defined by $\boldsymbol{Q} * \mathbb{T}=T_{\mathrm{ij} \ldots 1}\left(\boldsymbol{Q}_{\mathrm{ei}}\right) \otimes\left(\boldsymbol{Q}_{\mathrm{ej}}\right) \otimes \ldots \otimes\left(\boldsymbol{Q}_{\mathrm{el}}\right)$. The product $\boldsymbol{Q} * \mathbb{T}$ can be interpreted as the rotation of the tensor $\mathbb{T}$ by the orthogonal tensor $\boldsymbol{Q}$. For the analytical expression of the first-order bounds but also of other homogenization methods for cubic crystal aggregates, the interested reader is referred to, e.g., [2].

Self-consistent estimate. The elementary item of the self-consistent method is the single inclusion solution by Eshelby. In the self-consistent sense, every constituent of the heterogeneous material is embedded as a single inclusion into a matrix material, which has the effective properties of the composite [3]. By this particular choice of matrix material properties, the method has an inherently implicit character. By the introduction of a homogeneous reference medium with stiffness tensor equal to the effective medium stiffness $\overline{\mathbb{C}}$ and under the assumptions of piecewise constant stress polarizations as well as statistical homogeneity, no long-range order correlation and ellipsoidal twopoint statistics [4], the effective elasticity tensors are given by

$$
\left\langle\mathbb{C}\left(\mathbb{I}^{S}+\overline{\mathbb{P}} \delta \mathbb{C}\right)^{-1}\right\rangle\left\langle\left(\mathbb{I}^{S}+\overline{\mathbb{P}} \delta \mathbb{C}\right)^{-1}\right\rangle^{-1}, \quad \overline{\mathbb{S}}=\overline{\mathbb{C}}^{-1}
$$


In Eq. 4, the deviation of the local stiffness from the effective stiffness tensor is denoted by $(\boldsymbol{x})-\overline{\mathbb{C}}$ with $\mathbb{C}(\boldsymbol{x})=\mathbb{C}(\boldsymbol{Q}(\boldsymbol{x}))=\boldsymbol{Q}(\boldsymbol{x}) \star \tilde{\mathbb{C}}$ and $\overline{\mathbb{P}}$ is an Eshelby-like microstructural tensor [4], which is defined by

$$
\overline{\mathbb{P}}(\overline{\mathbb{C}})=\frac{1}{4 \pi \operatorname{det}(\boldsymbol{A})} \int_{n} \overline{\mathbb{H}}(\boldsymbol{n})\left(\boldsymbol{n} \cdot \boldsymbol{A}^{-\mathrm{T}} \boldsymbol{A}^{-1} \boldsymbol{n}\right)^{-3 / 2} \mathrm{~d} n
$$

The acoustic tensor $\bar{K}$ and the tensor $\overline{\mathbb{H}}$ are given by the relations

$$
\bar{H}_{i j k l}=\bar{N}_{i k} n_{j} n_{l}, \quad \bar{N}=\bar{K}^{-1}, \quad \bar{K}_{i k}=\bar{C}_{i j k l} n_{j} n_{l} .
$$

In Eq. 5, $\boldsymbol{A}$ accounts for the ellipsoidal two-point statistics, where the special case of $\boldsymbol{A}=\boldsymbol{I}$ (I is the second-order identity tensor) corresponds to an isotropic two-point correlation function, i.e., spherical grains. The estimate (Eq.4) is also self-consistent in the sense that it delivers the same result irrespective of whether stiffnesses or compliances are considered for the averaging scheme.

\section{Finite element based full-field simulations}

A finite element model of a polycrystalline sheet metal has been generated using periodic Voronoi tessellations. By using isotropic and anisotropic point seeds, we are able to study the effect of grain morphology on the macroscopic elastic behavior. The sheet metal with its crystalline structure has been discretized by finite elements using a tetrahedron-based mesh generator [5] which assures a periodic mesh on opposing faces (Fig. 2). Periodic displacement boundary conditions have been applied to the sheet metal to determine the effective stiffness tensor of the crystalline aggregate by using the well-known procedure of applying six standard load cases to the finite element model.

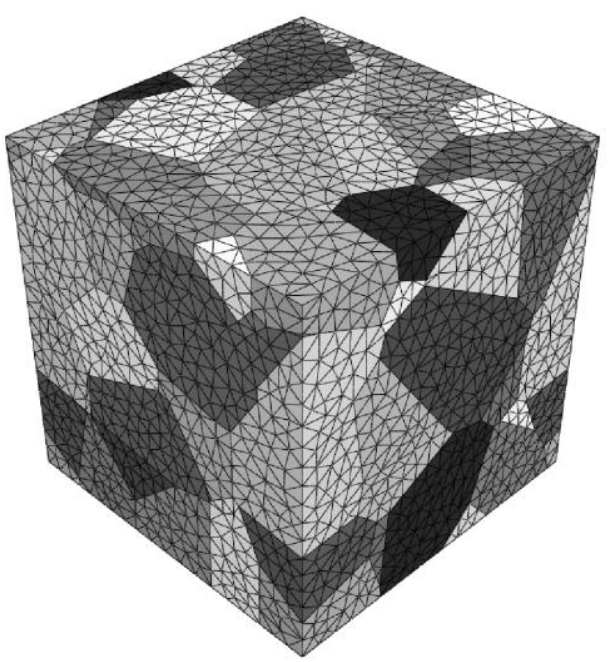

Fig. 2: Periodic finite element mesh for an aggregate of 50 grains for $\lambda=1$ ( $0 \%$ thickness reduction) 


\section{Results}

A sheet metal made of copper has been investigated to identify the influence of grain morphology and crystallite orientation distribution on Young's modulus in the sheet metal plane. Therefore, 50 random grain orientations out of the generated crystallographic texture by a Taylor simulation have been assigned to the finite element model. Furthermore, the same grain orientations were used to determine the Voigt and Reuss bounds as well as the self-consistent estimate of the effective stiffness tensor. Fig. 3 (left) shows the influence of the crystallographic texture $(50 \%$ thickness reduction: $\lambda=2$ ) on Young's modulus in the sheet metal plane. All methods (simple bounds, selfconsistent estimate and FE result) show a strong oscillation of Young's modulus in the sheet metal plane (25-30 GPa). The simple bounds represent boundaries for the real feasible material behavior, but they largely deviate from each other. The large gap between the bounds (30 GPa) results from the high elastic anisotropy of copper and would be much less pronounced in, e.g., aluminum. Furthermore, there is a very good agreement of the self-consistent estimate and the finite element result in the whole sheet metal plane, although the self-consistent method (and also the simple bounds) actually does not apply here, since the microstructure with 50 grains is not statistically homogeneous. Additionally, Fig. 3 (right) shows the influence of morphological texture on the Young's modulus estimate by using the self-consistent method. By keeping the crystallographic texture fixed and only varying the shape of the grains (aspect ratios $\lambda=1,2$ and 10), the influence of the morphology is clearly visible and, therefore, non-negligible. The grain morphology effect cannot be represented by the Voigt and Reuss bounds since they only incorporate the one-point correlation of the microstructure.
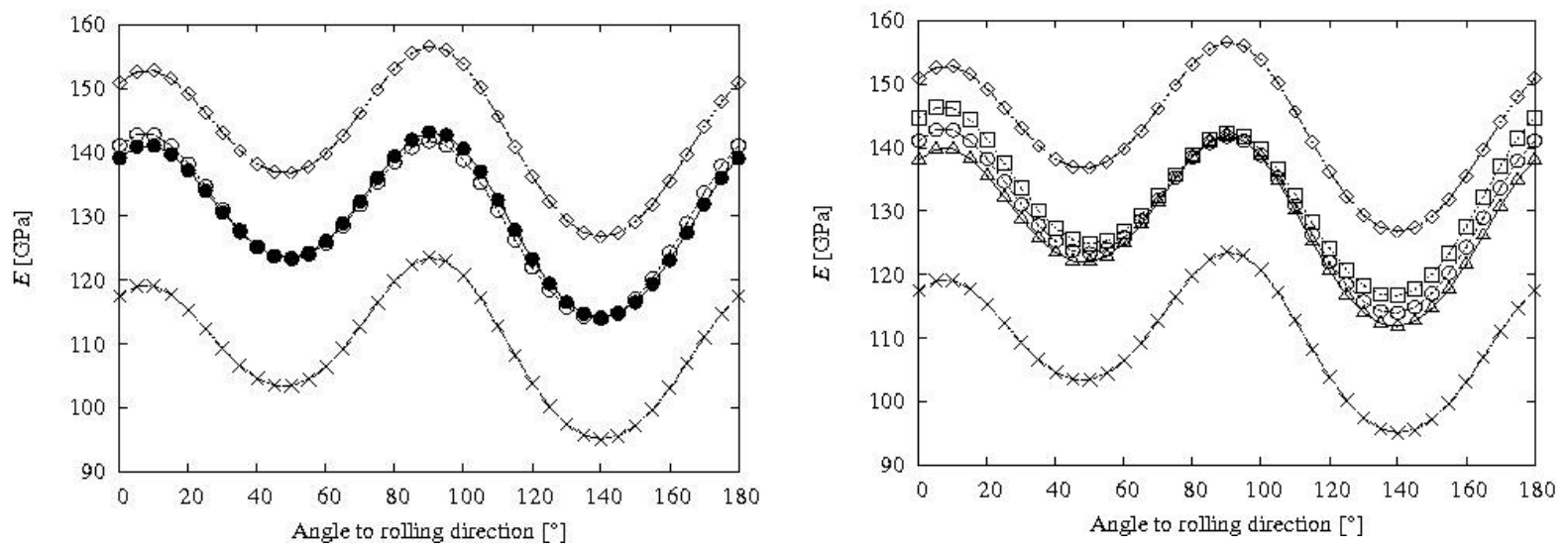

Fig. 3: left: Young's modulus in the sheet metal plane for $50 \%$ thickness reduction: Voigt $(\diamond)$ and Reuss $(\times)$ bounds, finite element result $(\bullet)$, self-consistent estimate $(0)$; right: Influence of grain morphology: self-consistent estimate for grain shapes with $\lambda=1(\triangle), \lambda=2(\circ)$ and $\lambda=10(\square)$.

\section{References}

[1] G.I. Taylor: Plastic strain in metals, J. Inst. Metals Vol. 62 (1938), p. 307-324.

[2] T. Böhlke, K. Jöchen, O. Kraft, D. Löhe and V. Schulze: Elastic properties of polycrystalline microcomponents, Mech. Mater. Vol. 42 (2010), p. 11-23.

[3] E. Kröner: Berechnung der elastischen Konstanten eines Vielkristalls aus den Konstanten des Einkristalls. Z. Phys. Vol. 151 (1958), p. 504-518.

[4] J.R. Willis: Bounds and self-consistent estimates for the overall properties of anisotropic composites. J. Mech. Phys. Solids Vol. 25 (1977), p. 185-202.

[5] F. Fritzen, T. Böhlke and E. Schnack: Periodic three-dimensional mesh generation for crystalline aggregates based on Voronoi tessellations. Comput. Mech. Vol. 43 (2009), p. 701-713. 